\title{
Ultrastructural and Immunofluorescent Analysis of Lithium Effects on Autophagy in Hepatocellular Carcinoma Cells
}

\author{
Iuliia Taskaeva ${ }^{1}$, Nataliya Bgatova ${ }^{2}$
}

${ }^{1}$ Researcher, laboratory of Ultrastructural research, Research Institute of Clinical and Experimental Lymphology - Branch of the Institute of Cytology and Genetics, Siberian Branch of Russian Academy of Sciences Timakova str., 2630060, Novosibirsk, Russia. ${ }^{2}$ Doctor of biological sciences, professor, Head of laboratory of Ultrastructural research, Research Institute of Clinical and Experimental Lymphology - Branch of the Institute of Cytology and Genetics, Siberian Branch of Russian Academy of Sciences Timakova str., 2630060, Novosibirsk, Russia.

\begin{abstract}
Background: Hepatocellular carcinoma (HCC) is one of the most malignant cancers worldwide. The role of autophagy in the HCC development and progression is controversial and are still not well known. It has been shown that lithium induces autophagy and apoptosis, affects on proliferation and survival of various human malignancies. In this study we estimated lithium`s effects on autophagy in HCC in vivo. Materials and methods: Subcellular components and autophagic vacuoles were analyzed by transmission electron microscopy. The number of cells with LC3 and LAMP1 punctate was analyzed by double-immunofluorescence staining. Data are presented as mean \pm standard deviation (SD). Mann-Whitney nonparametric tests were used to assess statistically significant differences at $\mathrm{P}<0.05$. Results: Lithium carbonate increased the numerical and volume density of autophagic vacuoles and autophagy marker LC 3 beta. Therefore, lithium carbonate promotes enlarged autophagic vacuoles formation in HCC cells in vivo. Conclusion: Extended and unresolved autophagy induces cancer cell death by stimulating autophagic cell death. Thus, lithium-mediated autophagy can be an attractive approach in HCC chemotherapy.
\end{abstract}

Keywords: Hepatocellular carcinoma- lithium carbonate- autophagy- electron microscopy- immunofluorescence

Asian Pac J Cancer Biol, 3 (3), 83-87

\section{Introduction}

Autophagy is the process that allows cells to isolate and degrade subcellular components in lysosomes and/or vacuoles and subsequently process them for cell metabolism. The role of autophagy in cancerogenesis is controversial. On one hand, autophagy can promote the survival of cancer cells under stress conditions or the deprivation of nutrients. On the other hand, autophagy acts as tumor suppressor, by stimulating autophagic cell death (ACD). ACD is a form of cell death which characterized by the large-scale autophagic vacuolization of the cytoplasm [1] and several studies have suggested that extended and unresolved autophagy promotes cancer cell death by ACD [2-3]. Hepatocellular carcinoma (HCC) is one of the most malignant and deadly cancers worldwide [4-5]. It is considered to the role of autophagy in the HCC development and progression is also controversial and
Submission Date: 07/14/2018 Acceptance Date: 10/13/2018

still not well known [6-8]. Recent studies reported that ACD promotion can enhance the response cancer cells to chemotherapy [9-11]. Therefore, stimulation of autophagy can be an attractive approach in HCC chemotherapy. Lithium induces autophagy and apoptosis, affects on proliferation and survival of tumor cells [12-14], and thus, can ranks as an antitumor agent for HCC therapy. In this study we estimated lithium's effects on autophagy in HCC population in vivo.

\section{Materials and Methods}

\section{Cell line and reagents}

Hepatocellular carcinoma-29 (HCC-29) cell line was obtained from the Institute of Cytology and Genetics, Siberian Branch, Russian Academy of Sciences [15]. Lithium carbonate was from "Novosibirsk rare metals plant" (Russia).

\section{Corresponding Author:}

Dr. Iuliia Taskaeva

Department Researcher, laboratory of Ultrastructural research, Research Institute of Clinical and Experimental Lymphology - Branch of the

Institute of Cytology and Genetics, Siberian Branch of Russian Academy of Sciences Timakova str.,2630060, Novosibirsk, Russia.

Email: inabrite@yandex.ru 


\section{Animals and tumor induction}

Animal testing was performed in accordance with Directive 2010/63/EU. Mice were maintained at a constant room temperature $\left(23{ }^{\circ} \mathrm{C}\right)$ with a natural day/night light cycle in a conventional animal colony. Standard laboratory food and water were provided. Before experiments, the mice were afforded an adaptation period of at least 10 days. Male CBA mice of 6-8 weeks of age, with weights of 18-20 g were used in experiment. For tumor induction, $1 \cdot 10^{6}$ HCC-29 cells were transplanted into the abdominal cavity. After 10 days, ascitic fluid was removed and $2 \cdot 10^{6} \mathrm{HCC}-29$ cells suspended in $100 \mu \mathrm{L}$ of PBS were injected into the right thigh muscle. Mice were randomly divided into three experimental groups (five mice in each). All treatments were delivered in $100 \mu \mathrm{L}$ volumes given intramuscularly along periphery of the tumor every day. Experimental groups were as follows: the mice with intact tumor (Control); $0.9 \%$ saline (S) and $20 \mathrm{mM}$ lithium carbonate (LC). Lithium was given in sterile $0.9 \%$ saline. All drugs were injected using aseptic techniques to avoid contamination. All mice were euthanized by cervical dislocation under overdose of anesthesia on the 23rd day of the experiment.

\section{Transmission electron microscopy (TEM)}

Tumor tissue was fixed with a $4 \%$ paraformaldehyde and then incubated with $1 \%$ osmium tetroxide $\left(\mathrm{OsO}_{4}\right)$ at $4^{\circ} \mathrm{C}$ for $1 \mathrm{~h}$. The tumor tissue was then incubated with $1 \%$ uranyl acetate after which it were further processed at the JEM 1400 electron microscope (JEOL, Japan). For each group $100 \mathrm{HCC}$ cells, containing nucleus and distinct uninterrupted cell membrane were randomly selected. For each cell volume (Vv) and numerical (NA) densities of autophagic vacuoles (AV) and lysosomes were counted and analyzed at $\times 8000$ magnification using ImageJ software (National Institutes of Health, Bethesda, MD). Identification of autophagic structures and quantification of autophagy by TEM morphometry were estimated as described in autophagy monitoring guidelines [16]. Initial autophagic vacuoles (AVi) or autophagosomes had double membrane, visible as two parallel membrane layers separated by electron-translucent cleft. Degradative autophagic vacuoles (AVd) or autolysosomes had one limiting membrane and contained heterogeneous electron dense cytoplasmic material at different stages of degradation. Lysosomes had high electron density and contained relatively homogeneous material.

\section{Immunofluorescent staining (IF-F)}

Frozen sections of tumor tissue $(15 \mu \mathrm{m})$ were analyzed by double-immunofluorescence staining using anti-LC3 beta $(1 / 200 ;$ ab48394, Abcam, UK) and anti-LAMP1 (1/200; ab25245, Abcam, UK) primary antibodies. Alexa Fluor 488-conjugated anti-rabbit IgG (1/200; ab150077, Abcam, UK) and Alexa Fluor 568-conjugated anti-rat IgG (1/200; ab175476, Abcam, UK) were used to detect the corresponding primary antibodies. Images were analyzed using an Axio Observer Z1 (Zeiss, Germany) fluorescence microscope at $\times 400$ final magnification.
Six fields per group were captured. The number of cells with LC3 and LAMP1 punctate staining was counted and analyzed using ImageJ software (National Institutes of Health, Bethesda, MD).

\section{Statistical analysis}

Data are presented as mean $(\mathrm{M}) \pm$ standard deviation (SD). Mann-Whitney nonparametric tests were used to assess differences by statistical package Statistica 6.0 (StatSoft, USA). Statistically significant differences were considered at $\mathrm{P}<0.05$.

\section{Results}

TEM analyses of ultrastructural changes in HCC cells. The differences concerning the ultrastructural characteristics detected between control groups and LC group regarded the structural polymorphism tumor cells (nucleus and cytoplasm sizes, concentrations and differences of subcellular components) (Figure 1, A). Mitochondrial changes observed included swelling, cristae fragmentation and loss of matrix density (Figure 1, B). The cisternae of rough endoplasmic reticulum were limited and multiple free polysomes were resided in cytoplasm (Figure 1, B).

Lithium carbonate induced autophagic vacuoles formation in HCC-29 cells in vivo (Figure 2). Autophagic vacuoles varied in sizes: AVi were not abundant and contained different portions of the cytosol (Figure 3, A). AVd predominated among cell vacuoles and the highly heterogeneity in AVd sizes, shapes and luminal undigested material were noticed (Figure 3, B, C). Lysosomes were occasionally distributed throughout the cytoplasm and had a homogeneous electron-dense material (Figure 3, D). The number of cells that had AVi and/ or AVd was higher after lithium introduction - $59 \%$ (versus Control - $48 \%$ and $\mathrm{S}-35 \%$ ) (Figure 3, E). TEM analysis of autophagic vacuoles revealed a marked increase in the volume $(\mathrm{Vv})$ and numerical (NA) densities in LC group (Figure 3, F, G). The numerical density of AVd in the LC group also was more abundant (Figure 3, H),

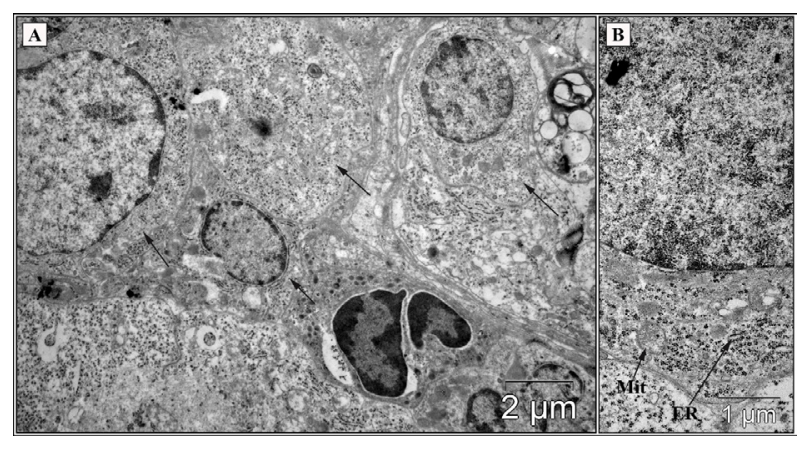

Figure 1. Ultrastructural Organization of HCC-29 Cells (TEM). The heterogeneity of tumor cells was backed by differences between nucleus and cytoplasm sizes, concentrations of subcellular components (A). Mitochondrial changes (B) included swelling, cristae fragmentation and loss of matrix density (Mit); the cisternae of rough endoplasmic reticulum were limited (ER). Scale bars as indicated. 


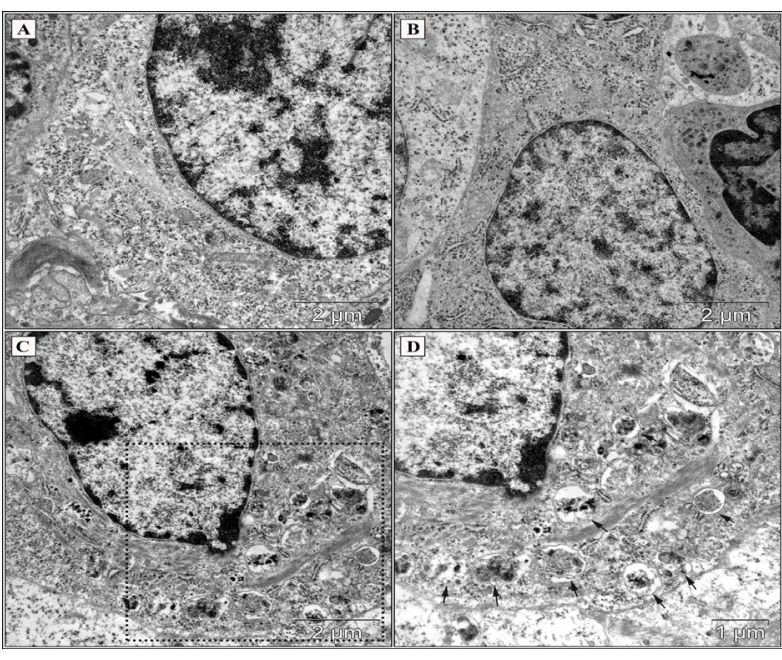

Figure 2. Morphology of Hepatocellular Carcinoma Cells (TEM) in the Control Group (A), Saline Group (B) and after Introduction of $20 \mathrm{mM}$ Lithium Carbonate at the Periphery of the Tumor (C, D). Arrows indicate multiple degradative autophagic vacuoles containing intracellular undifferentiated components (C - low magnification; D - high magnification from black box in C). Scale bars as indicated.

while $\mathrm{Vv}$ and NA of both AVi and lysosomes were comparable to the control.

Autophagy revealed in $\mathrm{HCC}$ cells by immunofluorescence. The expression of the autophagy markers LC3 beta and LAMP1 were assessed by immunofluorescence staining of frozen tumor tissue (Figure 4, A). IF-F results showed a significant increase of

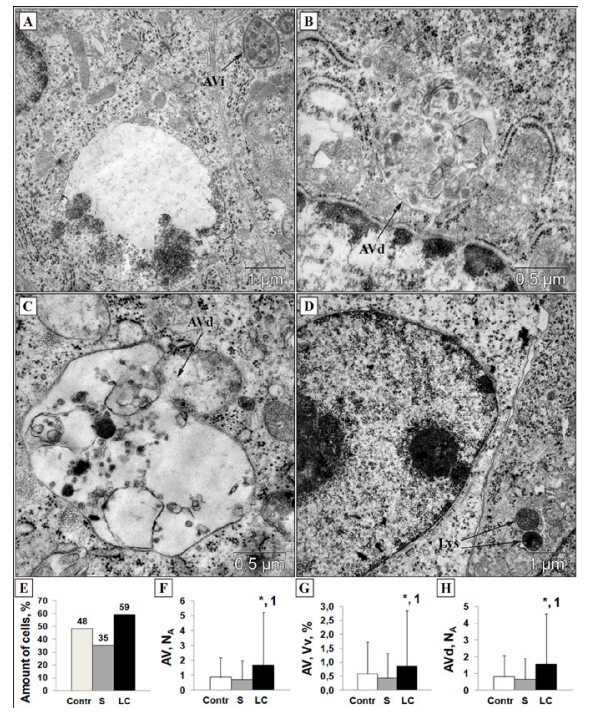

Figure 3. Ultrastructural Morphology of Initial Autophagic Vacuole (AVi, A), Degradative Autophagic Vacuoles (AVd, B and C) and Lysosomes (Lys, D) (TEM). Scale bars as indicated. Lithium carbonate increased cells with $\mathrm{AVi}$ and/or AVd (E); numerical (F) and volume (G) densities of autophagic vacuoles (AVi $+\mathrm{AVd})$; numerical density of AVd $(\mathrm{H})$. Contr - control; S saline; LC - lithium carbonate. Statistical analysis was carried out a Mann-Whitney U test $(* \mathrm{P}<0.05$ compared to control; ${ }^{1} \mathrm{P}<0.005$ compared to saline). Data were represented as mean $\pm \mathrm{SD}$. Electron microscopy images were analyzed using Image J software.

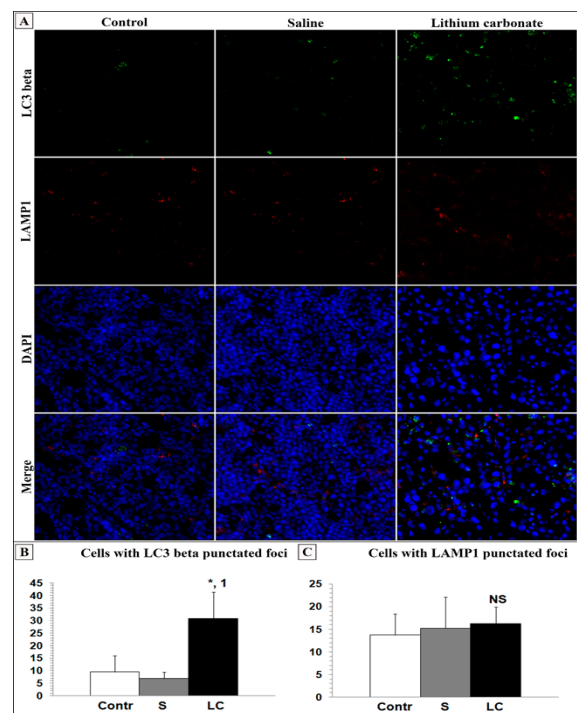

Figure 4. Double Immunofluorescent Staining (IF-F) Revealed Accumulation of LC3 (+) and LAMP1 (+) Vesicles in HCC-29 Cells in Vivo (magnification: $x$ 400). Lithium carbonate increased amount of cells with LC3 (A) and LAMP1 (B) punctated foci. Contr - control; $\mathrm{S}$ - saline; LC - lithium carbonate. Statistical analysis was carried out a Mann-Whitney $\mathrm{U}$ test $(* \mathrm{P}<0.05$ compared to control; ${ }^{1} \mathrm{P}<0.005$ compared to saline). NS - no significance. Data were represented as mean \pm SD. Fluorescent microscopy images were analyzed using Image J software.

LC3 beta (Figure 4, B) and a modest increase of LAMP1 (Figure 4, C) after lithium carbonate treatment. Taken together, these findings are indicative of lithium-mediated autophagy in HCC cells in vivo.

\section{Discussion}

Autophagy in cancer cells can have a dual role. It is known that autophagy promotes cancer cell survival under stress conditions, which confirmed by enhancement of autophagy in hypoxic regions of tumors, especially of solid type [17-18]; furthermore, autophagy can stimulates the resistance to antitumor therapy [19-20]. Alternatively, autophagy also acts as a tumor suppressor mechanism:

1. Selective degradation of mitochondria (mitophagy) reduces main sources of reactive oxygen species, which accelerates mutagenesis and cancerogenesis [17];

2. Autophagy promotes genomic stability by eliminating potentially damaged or genotoxic organelles, protein aggregates and autophagy substrate p62 (Sequestosome 1) $[21-22]$;

3. Autophagy participates in the cross-presentation of tumor associated antigens by MHC (major histocompatibility complex) molecules and stimulation of immunologic cell death, which enhanced antitumor immunosurveillance [22-26]. Taken together, all these evidence suggests the possible tumor suppressor role of autophagy.

In general, autophagy can be regulated by mTOR (mammalian target of rapamycin)-dependent and mTOR-independent signalling pathways [27]. Lithium enhances autophagy by modulating the phosphatidylinositol pathway, independently of the PI3K/AKT/mTOR pathway. 
Sarkar et al. [28] revealed that lithium induces autophagy by inhibiting inositol monophosphatase (IMPase), which result to decreased free inositol and mio-inositol-1,4,5triphosphate (IP3) levels. However, later Sade et al. [29] showed that elevated IP3 levels stimulate autophagy, and they proposed that lithium promotes IP3 accumulation which causing IP3 receptors (IP3Rs) desensitization and downregulation. A study by Vicencio et al. [30] has shown that IP3R formed a molecular complex with both Beclin 1 and Bcl-2, and disrupting this interaction led to Bcl-2-mediated autophagy inhibition; accordingly, lithium-mediated IP3Rs desensitization/downregulation reduces availability of IP3Rs, which would promote autophagy [29].

It has been shown that lithium is effective in inhibiting glioma [31], esophageal cancer [32], pancreatic cancer [33], colorectal cancer [34], leukemia [13-35] and other cancer cells; and several studies suggested the beneficial effects of lithium on cancer cell death and autophagy. O'Donovan et al. [36] have demonstrated that lithium-treated colorectal cells showed autophagosomes formation and vesicle accumulation. In addition, other study reported that treatment with $30 \mathrm{mM} \mathrm{LiCl}$ increased autophagic flux in colon cancer cells [37]. Furthermore, some studies have investigated lithium treatment of hepatocellular carcinoma. Erdal et al. [38] have provided evidence that lithium inhibits the growth of HCC cell lines, affects morphology, proliferation and cell cycle arrest in $\mathrm{HCC}$ cells. Interestingly, analysis of cell morphology in this study revealed significant morphological changes in HCC cells after lithium treatment in vitro: there was an increase in nucleus/cytoplasm ratios and multinuclear cell formation. Beurel et al. [39] revealed that lithium sensitized HCC cells to TRAIL-induced apoptosis. Taken together, these results indicate that lithium promotes autophagy in cancer cells and inhibits both growth and extension of tumor.

In this study we investigated effects of lithium on autophagy induction in HCC cells in vivo. Our results show a significant increase autophagic vacuoles following lithium treatment, revealed by TEM. Also, the punctuated LC3 and LAMP1 staining increased in HCC cells treated to 23 days of lithium. Collectively, our findings demonstrate that lithium carbonate promotes autophagy in HCC cells in vivo. In conclusion, further studies are required to complex monitoring autophagic flux and exploring lithium-mediated autophagy for sensitizing HCC cells to killing by chemotherapy.

\section{Funding}

This work was supported by the budgetary financing (project № 0324-2018-0006).

\section{Acknowledgements}

The authors are grateful to A.O. Solovieva for her assistance in preparing the cell line for transplantation and to V.V. Makarova for assisting in the experiment.

Authors' contributions
IT carried out the experimental part, performed TEM and IF-F, also images and experimental data analyses. NB carried out the experimental part and analyzed the experimental data. All authors participated in preparing the manuscript.

\section{Competing interests}

The authors declare no conflict of interests.

\section{References}

1. Kroemer, G. and Levine, B. Autophagic cell death: the story of a misnomer. Nature reviews. Molecular cell biology. 2008;9(12):1004-10.

2. Kondo, Y., Kanzawa, T., Sawaya, R. and Kondo, S. The role of autophagy in cancer development and response to therapy. Nature reviews. Cancer, 2005;5(9):726-34.

3. Janku, F., McConkey, D.J., Hong, D.S. and Kurzrock, R. Autophagy as a target for anticancer therapy. Nature reviews. Clinical oncology. 2011;8(9):528-39.

4. Best, J., Schotten, C., Theysohn, J.M., Wetter, A., Müller, S., Radünz, S., et al. Novel implications in the treatment of hepatocellular carcinoma. Annals of gastroenterology. 2017;30:23-32.

5. Hartke, J., Johnson, M. and Ghabril, M. The diagnosis and treatment of hepatocellular carcinoma. Seminars in diagnostic pathology. 2015;34:153-159.

6. Lee, Y.J. and Jang, B.K. The role of autophagy in hepatocellular carcinoma.

International journal of molecular sciences. 2015;16:26629-43.

7. Dash, S., Chava, S., Chandra, P.K., Aydin, Y., Balart, L.A. and $\mathrm{Wu}, \mathrm{T}$. Autophagy in hepatocellular carcinomas: from pathophysiology to therapeutic response. Hepatic medicine : evidence and research. 2016;8:9-20.

8. Liu, L., Liao, J.Z., He, X.X. and Li, P.Y. The role of autophagy in hepatocellular carcinoma: friend or foe. Oncotarget. 2017;8:57707-57722.

9. Washington, M.N., Suh, G., Orozco, A.F., Sutton, M.N., Yang, H., Wang, Y., et al. ARHI (DIRAS3)-mediated autophagyassociated cell death enhances chemosensitivity to cisplatin in ovarian cancer cell lines and xenografts. Cell death and disease. 2015. Available at: doi: 10.1038/cddis.2015.208.

10. Wang, Z., Liu, S., Ding, K., Ding, S., Li, C., Lu, J. et al. Silencing Livin induces apoptotic and autophagic cell death, increasing chemotherapeutic sensitivity to cisplatin of renal carcinoma cells. Tumor biology. 2016;37(11):15133-15143.

11. Zhang, C., Lei, J.L., Zhang, H., Xia, Y.Z., Yu, P., Yang, L. and Kong, L.Y. Calyxin Y sensitizes cisplatin-sensitive and resistant hepatocellular carcinoma cells to cisplatin through apoptotic and autophagic cell death via SCF $\beta$ TrCP-mediated eEF2K degradation. Oncotarget. 2017;8(41):70595-70616.

12. Sarkar, S., Ravikumar, B., Floto, R.A. and Rubinsztein, D.C. Rapamycin and mTOR-independent autophagy inducers ameliorate toxicity of polyglutamine-expanded huntingtin and related proteinopathies. Cell death and differentiation. 2009; 16:46-56.

13. Li, L., Song, H., Zhong, L., Yang, R., Yang, X.Q., Jiang, K.L. and Liu, B.Z. Lithium chloride promotes apoptosis in human leukemia NB4 cells by inhibiting glycogen synthase kinase-3 beta. International journal of medical sciences. 2015; 12:805-10.

14. Zassadowski, F., Pokorna, K., Ferre, N., Guidez, F., Llopis, L., Chourbagi, O., et al. Lithium chloride antileukemic activity in acute promyelocytic leukemia is GSK-3 and MEK/ERK dependent. Leukemia. 2015;29:2277-84.

15. Kaledin, V.I., Zhukova, N.A., Nikolin, V.P., Popova, N.A., 
Beliaev, M.D., Baginskaya, N.V., et al. Hepatocarcinoma-29, a metastasizing transplantable mouse tumor inducing cachexia. Bulletin of experimental biology and medicine. 2009;148:903-8.

16. Klionsky, D.J., Abdelmohsen, K., Abe, A., Abedin, M.J., Abeliovich, H., Acevedo Arozena, A., et al. Guidelines for the use and interpretation of assays for monitoring autophagy (3rd edition). Autophagy. 2016;12:1-222.

17. Ávalos, Y., Canales, J., Bravo-Sagua, R., Criollo, A., Lavandero, S. and Quest, A.F. Tumor suppression and promotion by autophagy. BioMed research international. 2014. Available at: doi: 10.1155/2014/603980.

18. Janji, B., Viry, E., Moussay, E., Paggetti, J., Arakelian, T., Mgrditchian, T., et al. The multifaceted role of autophagy in tumor evasion from immune surveillance. Oncotarget. 2016;7:17591-607.

19. Kimmelman, A.C. and White, E. Autophagy and tumor metabolism. Cell metabolism. 2017;25:1037-1043.

20. Lorente, J., Velandia, C., Leal, J.A., Garcia-Mayea, Y., Lyakhovich, A., Kondoh, H. and LLeonart, M.E. The interplay between autophagy and tumorigenesis: exploiting autophagy as a means of anticancer therapy. Biological reviews of the Cambridge Philosophical Society. 2018;93:152-165.

21. White, E., Mehnert, J.M. and Chan, C.S. Autophagy, metabolism, and cancer. Clinical cancer research: an official journal of the American Association for Cancer Research. 2015;21:5037-46.

22. Pietrocola, F., Bravo-San Pedro, J.M., Galluzzi, L. and Kroemer, G. Autophagy in natural and therapy-driven anticancer immunosurveillance. Autophagy. 2017;13:21632170.

23. Pan, H., Chen, L., Xu, Y., Han, W., Lou, F., Fei, W., et al. Autophagy-associated immune responses and cancer immunotherapy. Oncotarget. 2016;7:21235-46.

24. Hu, L., Jiang, K., Ding, C. and Meng, S. Targeting autophagy for oncolytic immunotherapy. Biomedicines. 2017. Available at: doi: 10.3390/biomedicines5010005.

25. Zhong, Z., Sanchez-Lopez, E. and Karin, M. Autophagy, inflammation, and immunity: a troika governing cancer and its treatment. Cell. 2016;166:288-298.

26. Li, C.J., Liao, W.T., Wu, M.Y. and Chu, P.Y. New insights into the role of autophagy in tumor immune microenvironment. International journal of molecular sciences. 2017. Available at: doi: 10.3390/ijms 18071566 .

27. Motoi, Y., Shimada, K., Ishiguro, K. and Hattori, N. Lithium and autophagy. American Chemical Society chemical neuroscience. 2014;5:434-42.

28. Sarkar, S., Floto, R.A., Berger, Z., Imarisio, S., Cordenier, A., Pasco, M., et al. Lithium induces autophagy by inhibiting inositol monophosphatase. The Journal of cell biology. 2005;170:1101-11.

29. Sade, Y., Toker, L., Kara, N.Z., Einat, H., Rapoport, S., Moechars, D., et al. IP3 accumulation and/or inositol depletion: two downstream lithium's effects that may mediate its behavioral and cellular changes. Translational psychiatry. 2016. Available at: doi: 10.1038/tp.2016.217.

30. Vicencio, J.M., Ortiz, C., Criollo, A., Jones, A.W., Kepp, O., Galluzzi, L., et al. The inositol 1,4,5-trisphosphate receptor regulates autophagy through its interaction with Beclin 1 . Cell death and differentiation. 2009;16:1006-17.

31. Fu, Y., Jiao, Y., Zheng, S., Liang, A. and Hu, F. Combination of lithium chloride and pEGFP-N1-BmK CT effectively decreases proliferation and migration of $\mathrm{C} 6$ glioma cells. Cytotechnology. 2016;68:197-202.

32. Gao, S., Li, S., Duan, X., Gu, Z., Ma, Z., Yuan, X., et al.
Inhibition of glycogen synthase kinase 3 beta (GSK3 $\beta$ ) suppresses the progression of esophageal squamous cell carcinoma by modifying STAT3 activity. Molecular carcinogenesis. 2017;56:2301-2316.

33. Wang, X., Luo, C., Cheng, X. and Lu, M. Lithium and an EPAC-specific inhibitor ESI-09 synergistically suppress pancreatic cancer cell proliferation and survival. Acta biochimica et biophysica Sinica. 2017;49:573-580.

34. Costabile, V., Duraturo, F., Delrio, P., Rega, D., Pace, U., Liccardo, R., et al. Lithium chloride induces mesenchymal to epithelial reverting transition in primary colon cancer cell cultures. International journal of oncology. 2015;46:1913-23.

35. Peixoto-da-Silva, J., Calgarotto, A.K, Rocha, K.R., PalmeiraDos-Santos, C., Smaili, S.S., Pereira, G.J.S., et al. Lithium, a classic drug in psychiatry, improves nilotinib-mediated antileukemic effects. Biomedicine \& pharmacotherapy. 2018;99:237-244.

36. O’Donovan, T.R., Rajendran, S., O’Reilly, S., O’Sullivan, G.C. and McKenna, S.L. Lithium modulates autophagy in esophageal and colorectal cancer cells and enhances the efficacy of therapeutic agents in vitro and in vivo. PLoS One. 2015. Available at: doi: 10.1371/journal.pone.0134676.

37. Trnski, D., Sabol, M., Gojević, A., Martinić, M., Ozretić, P., Musani, V., et al. GSK3 $\beta$ and Gli3 play a role in activation of Hedgehog-Gli pathway in human colon cancer Targeting GSK $3 \beta$ downregulates the signaling pathway and reduces cell proliferation. Biochimica et biophysica acta. 2015;1852(12):2574-84.

38. Erdal, E., Ozturk, N., Cagatay, T., Eksioglu-Demiralp, E. and Ozturk, M. Lithium-mediated downregulation of $\mathrm{PKB} / \mathrm{Akt}$ and cyclin $\mathrm{E}$ with growth inhibition in hepatocellular carcinoma cells. International journal of cancer. 2005;115:903-10.

39. Beurel, E., Blivet-Van Eggelpoël, M.J., Kornprobst, M., Moritz, S., Delelo, R., Paye, F., et al. Glycogen synthase kinase-3 inhibitors augment TRAIL-induced apoptotic death in human hepatoma cells. Biochemical pharmacology. 2009;77:54-65

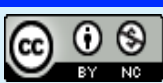

This work is licensed under a Creative Commons AttributionNon Commercial 4.0 International License. 